\title{
Assessments of Heavy Metal Concentration and Toxicity of Dust from a Parking Building in Bangkok
}

\author{
Boonyisa Suksomboon and Preprame Pattanamahakul
}

\begin{abstract}
Dust deposited in parking building is an important carrier of heavy metal contaminants and can contribute to urban runoff pollution. Dust deposited on parking floors (total of 10 floors) was collected from Chamchuri Square Building, Bangkok. The dust was separated into 5 particle sizes $(500-2000 \mu \mathrm{m}, 425-500 \mu \mathrm{m}, 212-425 \mu \mathrm{m}, 106-$ $212 \mu \mathrm{m},<106 \mu \mathrm{m})$ and each size was subjected to heavy metal analyses (Cd, $\mathrm{Co}, \mathrm{Fe}, \mathrm{Mn}, \mathrm{Ni}, \mathrm{Pb}$ and $\mathrm{Zn}$ ) by $\mathrm{HNO} 3 / \mathrm{HCl}$ extraction for total form and acetic acid extraction for exchangeable form. High level of Fe was observed in the total form extracts of all particle sizes while others such as $\mathrm{Zn}, \mathrm{Mn}$ and $\mathrm{Pb}$ showed different quantities in each particle size. However, $\mathrm{Zn}$ was found in most of exchangeable form extracts and others had no significant difference. Toxicity of the dust extract was assessed using duckweed (Lemna minor). Duckweed was exposed to five different concentrations of the extracts (from 37.5 to $600 \mathrm{mg} / \mathrm{ml}$ ) for 7 days, following the OECD test guideline 221. Growth rate and percent growth inhibition of duckweed were determined by calculating the changing number of fronds and frond areas. The dust extract had half inhibitory concentrations (IC50) of 263 and 350 $\mathrm{mg} / \mathrm{ml}$ for the number of fronds and frond areas, respectively. Therefore, dust deposited on parking floors should be monitored and disposed of properly to avoid ecological effects caused by its runoff.
\end{abstract}

Keywords - car park dust, heavy metal, toxicity testing, duckweed, frond number, frond area

\section{INTRODUCTION}

$\mathrm{D}$ UE to widespread urbanization in many countries, car parking building becomes a common place urban people frequently access. Automobile activities in the parking area can liberate many toxic substances, including carbon monoxide, VOCs and dust particles that carry PAHs [1] and heavy metals [2]. It is predicted that the level of heavy metals deposited in the dust particles corresponds with the activity of the car stopping. The higher frequency and distance of the car

Boonyisa Suksomboon Department of Environmental Science, Faculty of Science, Chulalongkorn University, Bangkok, Thailand

Preprame Pattanamahakul, Department of Environmental Science, Faculty of Science, Chulalongkorn University, Bangkok, Thailand stopping can contribute to the higher the heavy metal level in the dust. The structure of the parking building which is a semiclosed environment can promote the accumulation of the particulate in the area. Dust particles containing hazardous pollutants such as heavy metals and PAHs can pose health risk to users of the parking building and its runoff can generate adverse effects to organisms in the environment [3].

Lemna minor L. (Duckweed) is the most common species and widely accepted model for toxicity testing as model plant to assess ecological risks posed by various contaminants. It is listed in a test protocol of guidelines for the testing of chemicals: Lemna sp. Growth Inhibition Test [4] to study the number of leaves (fronds) and frond area. This guideline holds many advantages due to economically sustain, short experimental time and less complicated procedures compared to other toxicity tests.

Although the compositions of the dust in car parking structure have had a number of studies, there are a few studies on the toxicity of the dust. Moreover, with the congestion of traffic in Bangkok that would require longer time to get in and out of parking building and the differences of the structures of parking building may result in different types and compositions of the dust in the parking building. These compositions in the dust can give rise to adverse effects of health and ecosystem. Therefore, the objectives of this study were to determine the heavy metal content of the dust collected from a parking building in central Bangkok and evaluate the toxic effects of the dust extract on Lemna minor.

\section{MATERIALS AND METHODS}

\section{A. Sample collection and preparation}

Dust deposited on parking floors (total of 10 floors) was collected during October 2013 from Chamchuri Square building located in the central part of Bangkok, Thailand. The dust was then oven-dried at $40 \mathrm{oC}$ and sieved through a $2 \mathrm{~mm}$ sieve to remove large particles and stones. Then the remaining dust particle was separated into 4 size-fractions, i.e., less than $106 \mu \mathrm{m}, 106-212 \mu \mathrm{m}, 212-425 \mu \mathrm{m}$ and 500-2000 $\mu \mathrm{m}$.

\section{B. Analysis of heavy metal in parking dust}

For the analysis of the total form of heavy metal, the sieved dust samples $(2 \mathrm{~g})$ were digested with a mixture of $1: 365 \%$ 
HNO3 : $37 \% \mathrm{HCl}$. For the analysis of the exchangeable form, the sieved dust samples $(2 \mathrm{~g})$ were extracted with $0.11 \mathrm{M}$ acetic acid. Seven heavy metals, i.e., $\mathrm{Cd}, \mathrm{Co}, \mathrm{Fe}, \mathrm{Mn}, \mathrm{Ni}, \mathrm{Pb}$ and $\mathrm{Zn}$ were determined by inductively coupled plasma optical emission spectrometry (ICP-OES, JY 238 Ultrace Ultima, JY Jobin Yvon, Horiba).

\section{Toxicity assessment of parking dust extracts on duckweed}

For the preparation of the extract, distilled water was added to the dust and the mixture was shaken for 24 hours. The mixture was then filtered through a vacuum filtration to give a clear extract. Five concentrations of the dust extract were prepared, i.e., 600, 300, 150, 75 and $37.5 \mathrm{mg} / \mathrm{ml}$. Toxicity testing of the dust extracts was assessed using duckweed (Lemna minor) according to OECD 221 [4]. Each experiment was run in triplicate. In this study, frond number is the primary measurement variable and total frond area is also measured. To quantify the toxicity of the extract, growth of duckweed in the serial dilution of the extract was compared with that of the controls after 7 days of incubation and the concentration bringing about $50 \%$ inhibition of growth was determined and expressed as the $\mathrm{IC}_{50}$.

\section{Statistical Analysis}

All data were analyzed using Microsoft Excel and SPSS for windows (version 22). Arithmetical means \pm standard deviation ( $\mathrm{SD} ; \mathrm{n}=3$ ) were used to assess the contamination levels of heavy metals in the dust. Analysis of variance (ANOVA) and Dunnett's post test were used to determine the differences among the extract concentration and the frond number.

\section{RESULTS AND DISCUSSION}

\section{A. Size distribution of parking dust and heavy metals}

The parking dust was separated into 5 size-fractions, i.e., 500-2000 $\mu \mathrm{m}, 425-500 \mu \mathrm{m}, 212-425 \mu \mathrm{m}, 106-212 \mu \mathrm{m}$ and < $106 \mu \mathrm{m}$ (Figure 1 and Table 1). Based on the weight, most dust particles were between 500 and $2000 \mu \mathrm{m}$ which was $354.64 \mathrm{~g} / \mathrm{kg}$ of the whole dust $(=35.46 \%)$. The dust particles which were between 425 and $500 \mu \mathrm{m}$ had the lowest weight fraction $(70.12 \mathrm{~g} / \mathrm{kg}, 7.01 \%)$.

Figures 2 and 3 show the average concentrations of heavy metals in the total form and the exchangeable form, respectively, in the size fractions of the collected dust samples. High level of iron was observed in the total form extracts of all particle sizes while others such as zinc, manganese and lead showed different quantities in each particle size (Figure 2). However, zinc was found in most of exchangeable form extracts and others had no significant difference (Figure 3). The concentrations of the heavy metals identified in the dust decreased with the increasing particle size of the dust. Smaller particle size has more surface area for deposition of the heavy metals, therefore, the smaller particle has higher concentration of heavy metals. In addition, the amount of organic and clay mineral components that absorb metals easier are higher in the dust with the smaller particle size [5].

\section{B. Toxicity of Parking Dust}

Aqueous extract of the parking dust produced adverse effects to the duckweed when tested according to OECF test guideline 221. Figure 4 shows that the dust extract reduced both number of frond and area of frond at the concentrations of 150,300 and $600 \mathrm{mg} / \mathrm{ml}$. Percent inhibition of the growth was calculated by comparing the growth of duckweed in the series concentrations of the extract with that of the controls and the concentration that give $50 \%$ inhibition of growth was expressed as IC50. Figure 5 shows that the highest concentration of the dust extract $(600 \mathrm{mg} / \mathrm{ml})$ gave the highest percentage of growth inhibition representing $58.82 \%$ (number of fronds) and $54.41 \%$ (frond areas) while the lowest concentration of the dust extract illustrated the lowest percentage of growth inhibition (Figure 5). The dust extract had half inhibitory concentrations (IC50) of 263 and 350 $\mathrm{mg} / \mathrm{ml}$ for the number of fronds and frond areas, respectively. Heavy metals contamination in the runoff can cause harmful effects to living organisms [6]. Therefore, soluble heavy metals, in part, take responsible for the toxicity on duckweed exposed to the dust extract from the parking building.

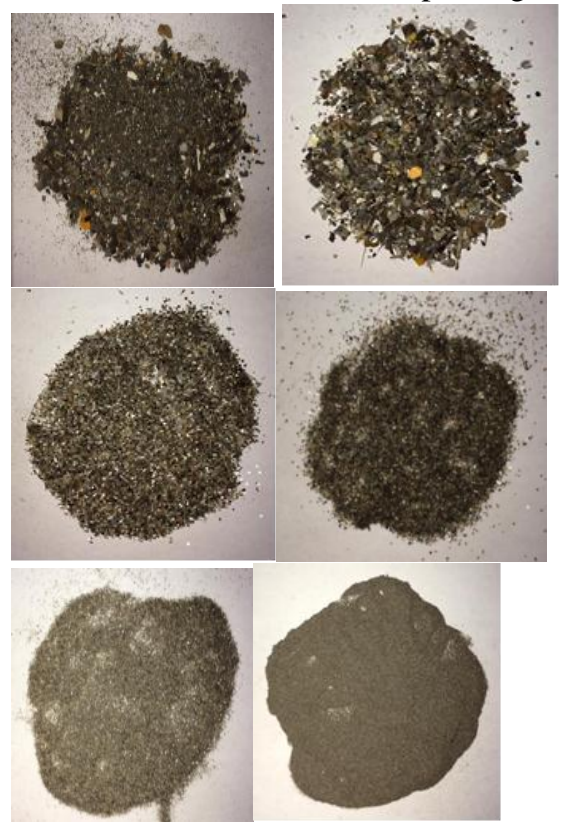

Fig. 1: Dust samples collected from car park of Chamchuri Square Building in Bangkok; (a) whole dust, (b) dust size 500-2000 $\mu \mathrm{m}$, (c) 425-500 $\mu \mathrm{m}$, (d) $212-425 \mu \mathrm{m}$, (e) 106-212 $\mu \mathrm{m}$ and (f) $<106 \mu \mathrm{m}$.

TABLE 1:

The WeIght AND WeIGHT \% OF DifFERENT Size Fractions CONSTITUTED PARKING DUST

\begin{tabular}{ccc}
\hline \hline Size $(\boldsymbol{\mu m})$ & Weight $(\mathbf{g} / \mathbf{k g}$ whole dust $)$ & Weight $\%(\boldsymbol{\%})$ \\
\hline $500-2000$ & 354.64 & 35.46 \\
$425-500$ & 70.12 & 7.01 \\
$212-425$ & 209.44 & 20.94 \\
$106-212$ & 220.05 & 22.01 \\
$<106$ & 145.75 & 14.58 \\
\hline \hline
\end{tabular}




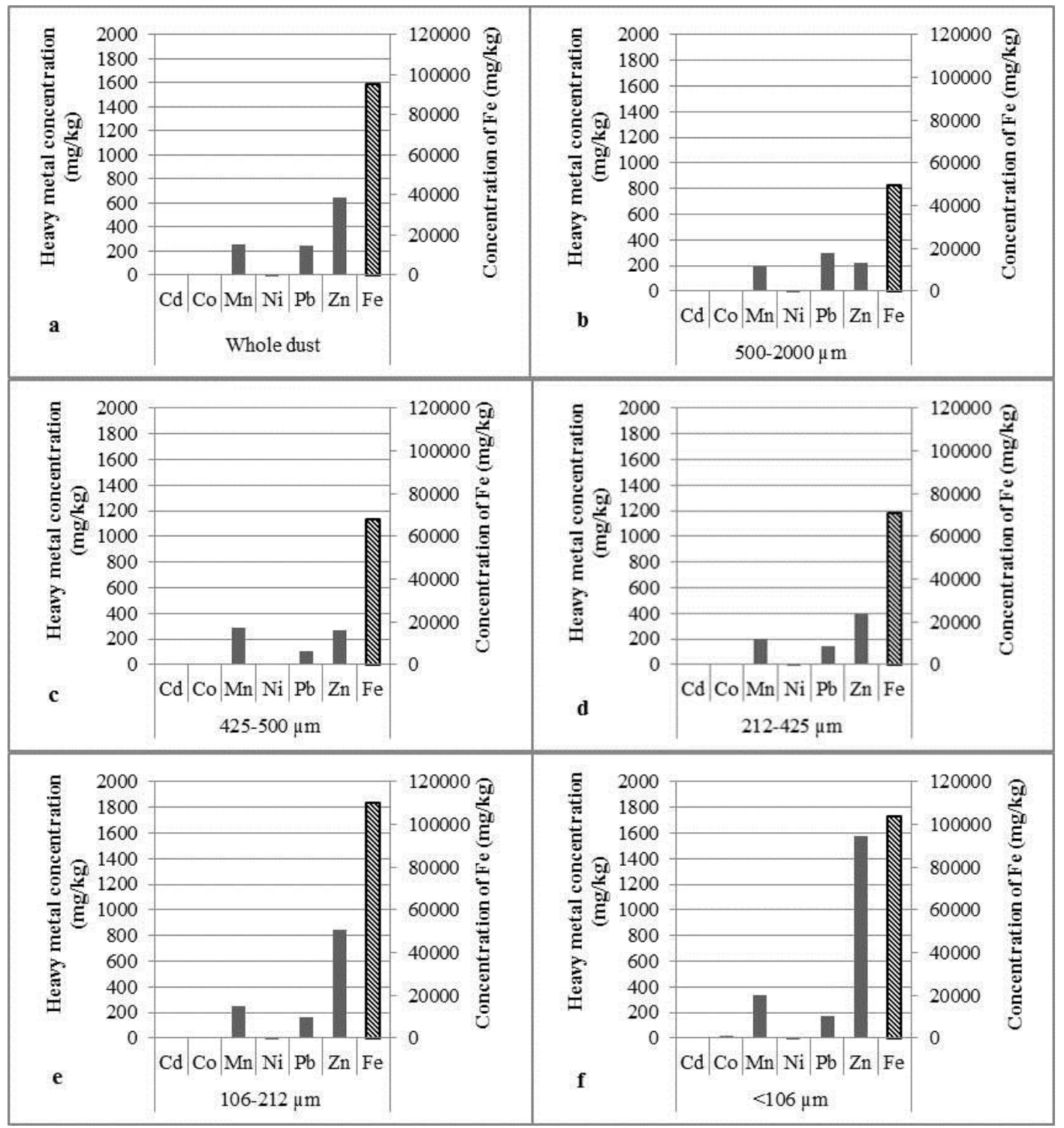

Fig. 2: Concentrations of heavy metals in the total form of the parking dust according to their size fractions; (a) whole dust, (b) size 500-2000 $\mu \mathrm{m}$, (c) $425-500 \mu \mathrm{m}$, (d) $212-425 \mu \mathrm{m}$, (e) $106-212 \mu \mathrm{m}$ and (f) $<106 \mu \mathrm{m}$. 


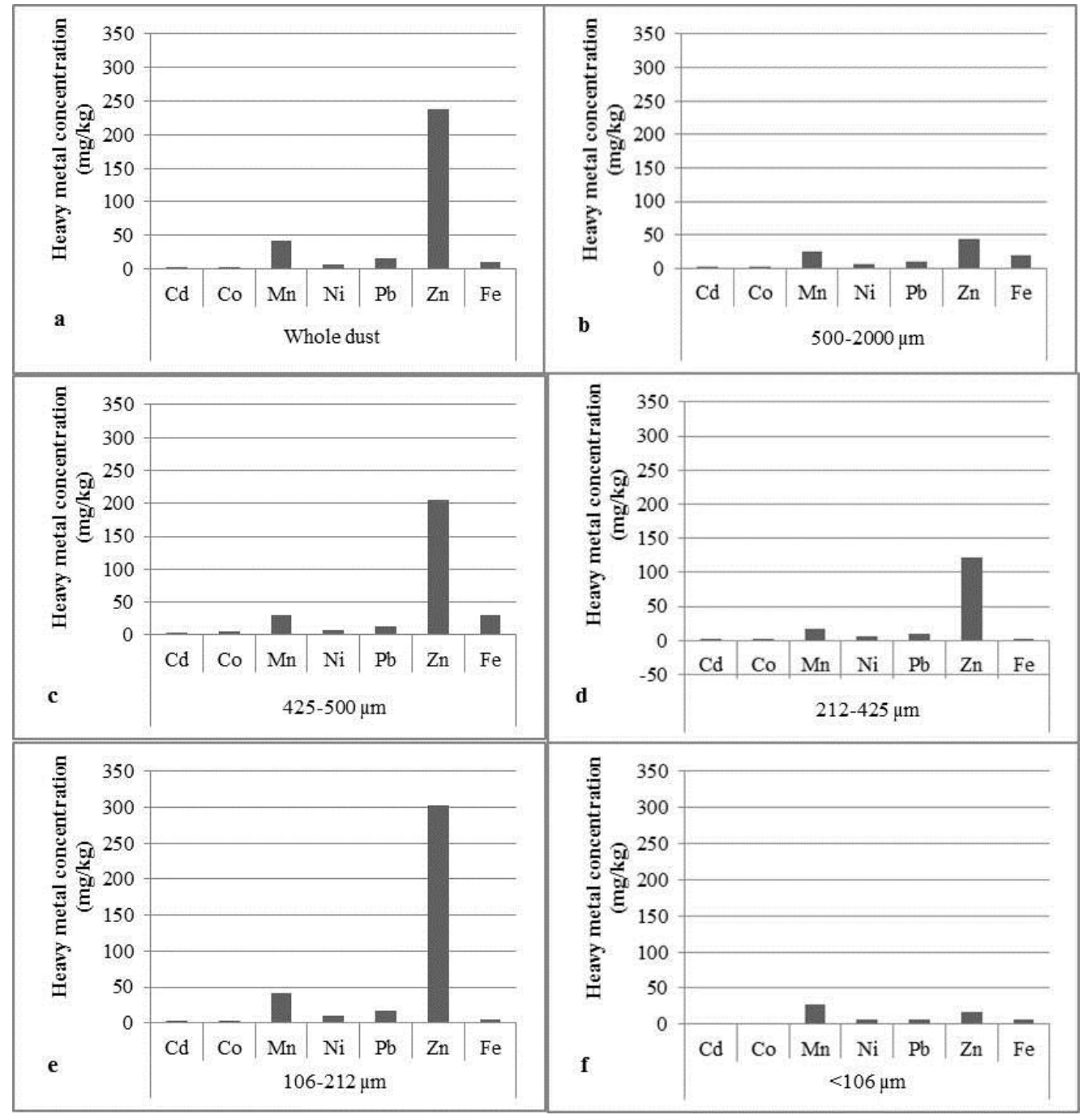

Fig. 3: Concentrations of heavy metals in the exchangeable form of the parking dust according to their size fractions; (a) whole dust, (b) size 500-2000 $\mu \mathrm{m}$, (c) 425-500 $\mu \mathrm{m}$, (d) 212-425 $\mu \mathrm{m}$, (e) 106-212 $\mu \mathrm{m}$ and (f) $<106 \mu \mathrm{m}$.
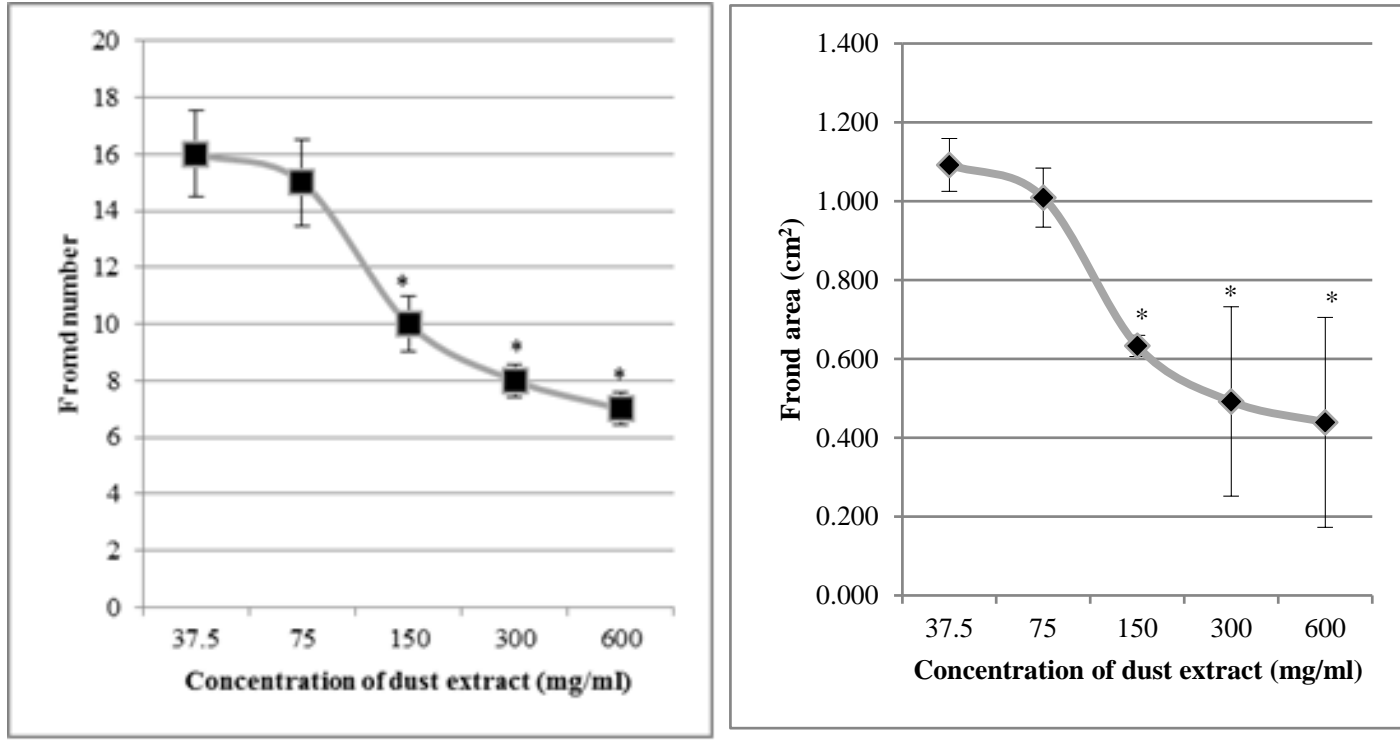

Fig. 4: Effects of parking dust extract solutions in the growth of (a) frond number and (b) frond area of duckweed evaluated over a period of 7 days. Mean $\pm \mathrm{SD}(\mathrm{n}=3)$. “*” indicates statistical differences between particular tested concentrations and control at $95 \%$ confidence level. 


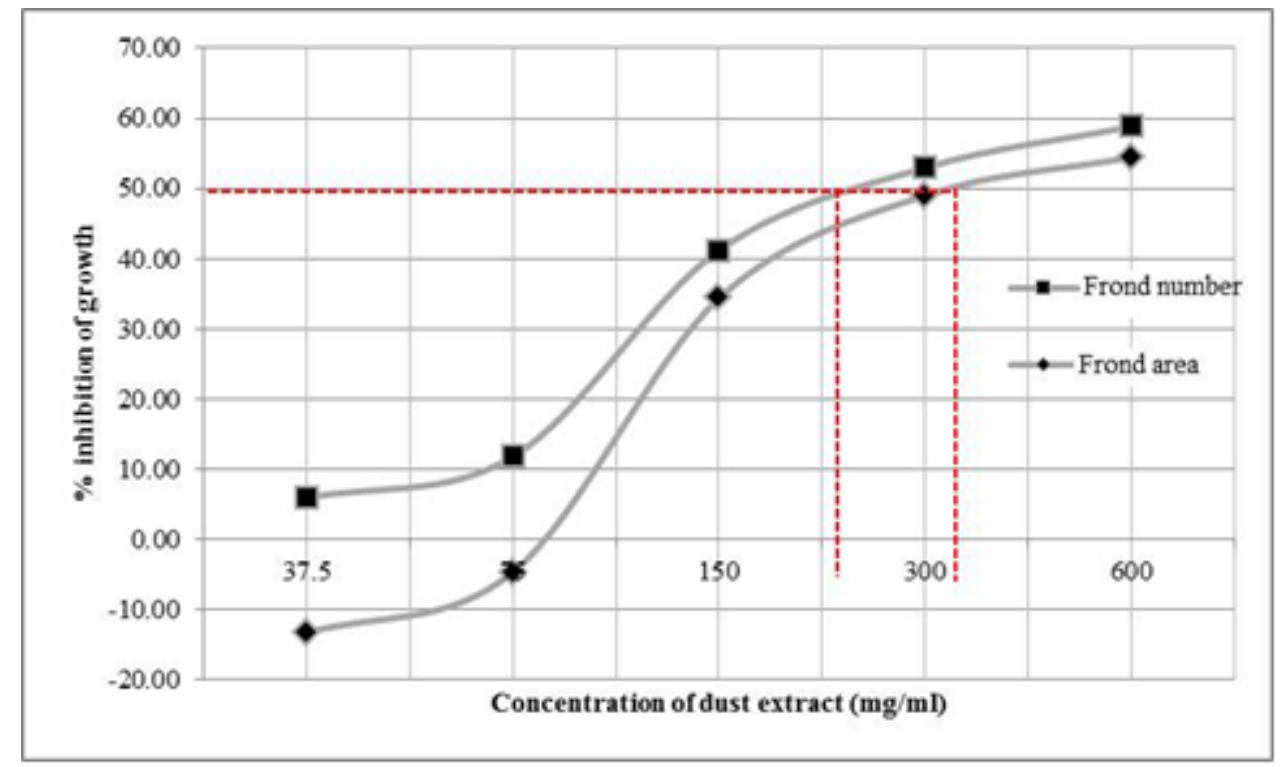

Fig. 5: Percentage of growth inhibition calculated from frond number and frond area.

\section{CONCLUSION}

In this study, dust collected from a parking building in Bangkok consisted of $\mathrm{Fe}>\mathrm{Zn}>\mathrm{Pb}=\mathrm{Mn}$, however, $\mathrm{Zn}$ was the most prominent exchangeable heavy metal as extracted in aqueous acetic acid. Aqueous extract of the parking dust produced adverse effects to the duckweed when tested according to OECF test guideline 221. The dust extract had IC50 of 263 and $350 \mathrm{mg} / \mathrm{ml}$ for the number of fronds and frond areas, respectively. Therefore, dust deposited on parking floors should be monitored and disposed of properly to avoid ecological effects caused by its runoff.

\section{V.ACKNOWLEDGEMENTS}

We would like to thank the Department of Environmental Science and the Faculty of Science, Chulalongkorn University for the supports of this Senior Project.

\section{REFERENCES}

[1] S.R. Kim, F. Dominici, and T.J. Buckley, "Concentrations of vehiclerelated air pollutants in an urban parking garage," Environmental Research, vol. 105, pp. 291-299, 2007.

[2] Y. Li, and R. Xiang, "Particulate pollution in an underground car park in Wuhan, China," Particuology, vol. 11, pp. 94-98, 2013.

[3] H. Watanabe, F. Nakajima, I. Kasuga, and H. Furumai, "Toxicity evaluation of road dust in the runoff process using a benthic ostracod Heterocypris incongruens," Science of The Total Environment, vol. 409, pp. 2366-2372, 2011.

[4] OECD, "OECD guidelines for the testing of chemicals, Test guideline 221, Lemna sp. growth inhibition test," OECD, Paris, France. 2006.

[5] P.R. Beckwith, J.B. Ellis, and D.M. Revitt, "Heavy metal and magnetic relationships for urban source sediments," Physics of the Earth and Planetary Interiors, vol. 42, pp. 67-75, 1986.

[6] D. Greenstein, L. Tiefenthaler, and S. Bay, "Toxicity of parking lot runoff after application of simulated rainfall," Archives of Environmental Contamination and Toxicology, vol. 47, pp. 199-206, 2004.

\section{Correspondence to}

Dr. Preprame Pattanamahakul, Department of Environmental

Science, Faculty of Science, Chulalongkorn University,

Phayathai Road, Bangkok 10330, Thailand, Tel: +66-2-218-5193,

Fax: +66-2-218-5180, E-mail: preprame.p@chula.ac.th 